\title{
Characterization of single semiflexible filaments under geometric constraints
}

\author{
S. Köster ${ }^{1, a}$, J. Kierfeld ${ }^{2, b}$, and T. Pfohl ${ }^{1, c}$ \\ 1 Max Planck Institute for Dynamics and Self-Organization, Bunsenstraße 10, 37073 Göttingen, Germany \\ 2 Max Planck Institute of Colloids and Interfaces, Science Park Golm, 14424 Potsdam, Germany
}

Received 7 October 2007 and Received in final form 13 February 2008

Published online: 21 April 2008 - (c) EDP Sciences / Società Italiana di Fisica / Springer-Verlag 2008

\begin{abstract}
Confinement effects on single semiflexible macromolecules are of central importance for a fundamental understanding of cellular processes involving biomacromolecules. To analyze the influence of confinement on the fluctuations of semiflexible macromolecules we study individual actin filaments in straight and curved microchannels. We experimentally characterize the segment distributions for fluctuating semiflexible filaments in microchannels as a function of the channel width. Moreover, the effect of channel curvature on the filament fluctuations is investigated. We find quantitative agreement between experimental results, Monte Carlo simulations, and the analytical description. This allows for determination of the persistence length of actin filaments, the deflection length, which characterizes the confinement effects, and the scaling exponents for the segment distribution of semiflexible macromolecules.
\end{abstract}

PACS. 87.16.Ka Filaments, microtubules, their networks, and supramolecular assemblies - 87.16.Ac Theory and modeling; computer simulation -82.37 .Rs Single molecule manipulation of proteins and other biological molecules

\section{Introduction}

In bottom-up approaches to cell mechanics [1-3] as well as in top-down approaches to nanotechnology for bioanalysis $[4,5]$, confinement effects on semiflexible macromolecules play a considerable role. Shape, motility, and proliferation of eukaryotic cells are regulated by the cytoskeleton, a meshwork of semiflexible biomacromolecules. In living cells, cytoskeletal filaments are confined in their own rather dense networks. Much effort is devoted to studies on networks of actin filaments, one of the key proteins of the cytoskeleton. The overall goal is to elucidate the mechanical and dynamic properties of these in vitro model systems and to exploit the results to obtain a better understanding of cell mechanics $[1,6,7]$. Although these investigations aim for an understanding of the collective behavior of cellular networks, they are strongly dependent on a profound knowledge about single filament dynamics confined by the surrounding macromolecules [8]. In vivo cell mechanics are more complex than these idealized

\footnotetext{
a Present address: Dept. of Physics/SEAS, Harvard University, 40 Oxford St., Cambridge, MA, USA.

b New permanent address: Lehrstuhl für Theoretische Physik I, Technische Universität Dortmund, 44221 Dortmund, Germany.

e-mail: thomas.pfohl@ds.mpg.de
}

model systems, since the microstructure and elasticity of the cytoskeleton are clearly heterogeneous and influenced by active processes such as motion of molecular motors and the polymerization and depolymerization of filaments. The living cell exploits confinement effects for structure formation, one fascinating example of which is the fibripositor (finger-like protrusions of the plasma membrane)mediated collagen fibril alignment in tendon [9].

In nanobioanalysis systems, transport and confinement of DNA and proteins within micro- and nanochannels are of particular importance [10-12]. The development of DNA-chip-based devices aims not only to detect and separate single DNA molecules, but also to sequence them on the single molecule level $[4,5]$. The devices are used to confine DNA on the nanometer scale and thus elongate genomic-length DNA. Confinement alters the statistical mechanical properties as well as the Brownian motion of the confined biomacromolecules [4,13-16]. Since in most nanodevices the widths of confining nanochannels $d$ are smaller than the persistence length $L_{P}$ of DNA $\left(L_{P} \sim 50 \mathrm{~nm}\right)$, the behavior of the DNA can only be described by a model of confined semiflexible macromolecules [17]. Confinement of DNA is also widely found in nature, the most prominent example being DNA compaction in the nucleus. The wrapping of the DNA around core particles in chromatin leads to confinement and simultaneous bending of the macromolecules $[18,19]$. 
In this work we study the Brownian dynamics and mechanical properties of actin filaments, which are confined in microchannels of different geometries. Apart from being a biologically relevant system, actin is known for its reliability as an experimental model system for semiflexible macromolecules. The width of the channels $d$ and the filament contour length $L$ as well as the persistence length $L_{P}$ are on the order of a few micrometers, which enables direct observation and visualization of fluorescently labeled macromolecules by optical microscopy. Typical radii of curvature of the channels are comparable to or larger than the persistence length $L_{P}$ and similar to typical curvatures of confining "tubes" in entangled actin solutions [8]. We characterize the segment distributions of fluctuating actin filaments as a function of the channel width as well as the influence of a curvature on the thermal fluctuations. By combining experimental, modeling, and analytical approaches, we provide a complete analysis of semiflexible filament behavior under geometric constraints on the single molecule level.

\section{Experimental methods}

Rhodamine labeled G-actin (Cytoskeleton, Denver, USA) is polymerized, stabilized using phalloidin, and diluted to a final monomer concentration of $70 \mathrm{nM}$. The detailed procedure is described in reference [13]. The dilute polymer solution is injected into microchannels which are fabricated using standard soft lithography techniques [20,21]. The depth $h$ of the PDMS microchannels for all experiments presented here is between 0.9 and $1.4 \mu \mathrm{m}$. We use parallel straight channels with widths of $d=1.5,4.0,5.8$, and $9.8 \mu \mathrm{m}$, and curved channels with different radii of curvature $(13.5-143.5 \mu \mathrm{m})$ and a width of $d=2.5 \mu \mathrm{m}$ (see Fig. 1). The microchannels are tightly sealed with glass cover slips. This allows for high-resolution fluorescence microscopy (see Fig. 2a) using an Olympus BX61 fluorescence microscope equipped with a $75 \mathrm{~W}$ xenon lamp and a $100 \times$ Plan Apochromat oil-immersion objective. Exposure times for the image sequences are $100 \mathrm{~ms}$. Examples of snapshots are shown in Figure 2a. Due to the small channel depth and the projection of the filament contour into the focal plane of the microscope, we observe F-actin in a quasi-2D geometry [22]. The channel walls are coated with bovine serum albumin (BSA) to avoid additional interactions of the actin filaments and the channel walls.

To obtain the tangent vectors $\mathbf{t}(s)$, the microscopy images are binarized and skeletonized to a one-pixel-line using commercial image processing software (Image-Pro Plus, AnalySIS, MATLAB). A cubic spline fit is applied to the one-pixel-line and an arclength reparametrization of the continuous line is obtained by dividing it into tangent vectors of equal length. The correlation function of the corresponding unit tangent vectors as a function of their arclength separation $l$ is calculated from the average $\langle\cos \theta(l)\rangle \equiv\langle\mathbf{t}(s) \cdot \mathbf{t}(s+l)\rangle$ of their scalar products $[13,14]$, where the thermodynamic average is performed by an average in time over all recorded conformations. In addition, we perform a spatial average over all points $s$ within the

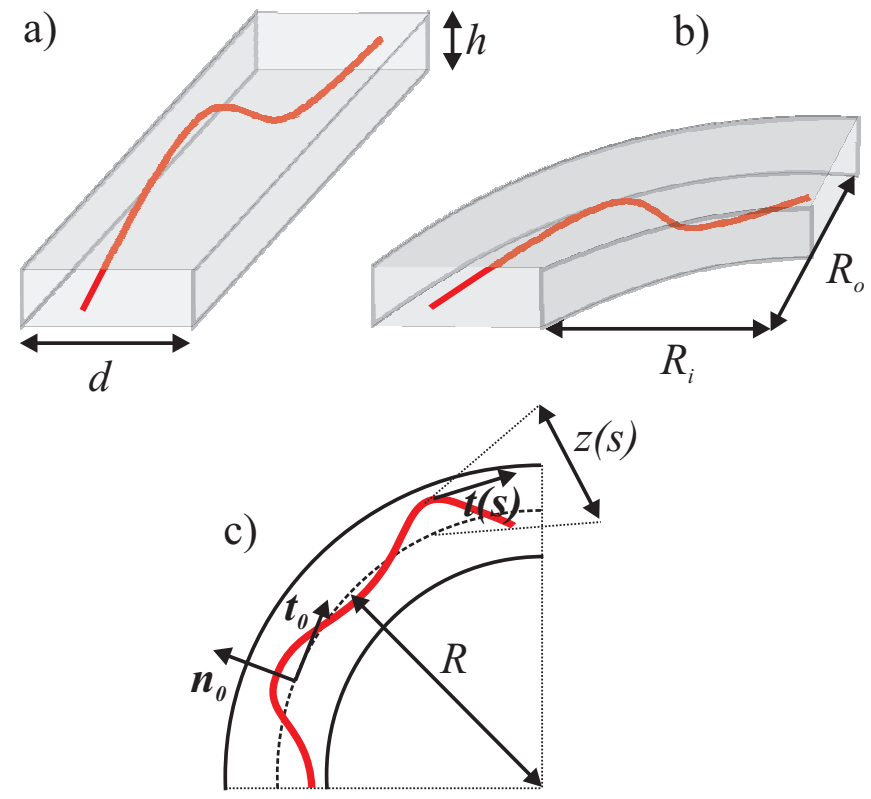

Fig. 1. Schematic representation of confined semiflexible filaments in different channel geometries. a) Straight channels. b) Curved channels. c) Parameters used to describe curved channels.

same image. To further improve the statistics of our results, we also average the data of several filaments where the same experimental parameters have been applied. In this case additional weighting by the filament length and number of recorded conformations is taken into account.

To obtain the segment distribution in straight channels, we rotate all images containing the one-pixel-lines such that the channel direction is exactly horizontal. We integrate the intensity over the full filament length along the direction of the channels, average the values over all recorded conformations of a filament, normalize the result by the length of the filament, mirror and average the data at the center line of the channel to improve the statistics. Results are plotted against the $z$-coordinate perpendicular to the channel, where we choose $z=0$ and $z=d$ as the positions of the channel walls.

\section{Theoretical model}

\subsection{Tangent correlations in curved channels}

We have introduced an analytical description of tangent correlations of fluctuating semiflexible filaments in straight channels under consideration of the apparent broadening of the filaments' contour in microscopy images [14]. For long and weakly bent filaments, we find the following scaling form for the tangent correlations:

$$
1-\langle\cos \theta(l)\rangle \approx \frac{\lambda}{L_{P}} \mathcal{C}\left(\frac{l}{\lambda}\right)
$$

with a shape function

$$
\mathcal{C}(x)=\frac{1}{2 \sqrt{2}}\left(\cos \left(\frac{\pi}{4}\right)-\cos \left(\frac{\pi}{4}+x\right) e^{-x}\right) .
$$


a)

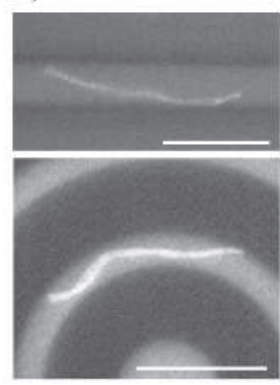

b)

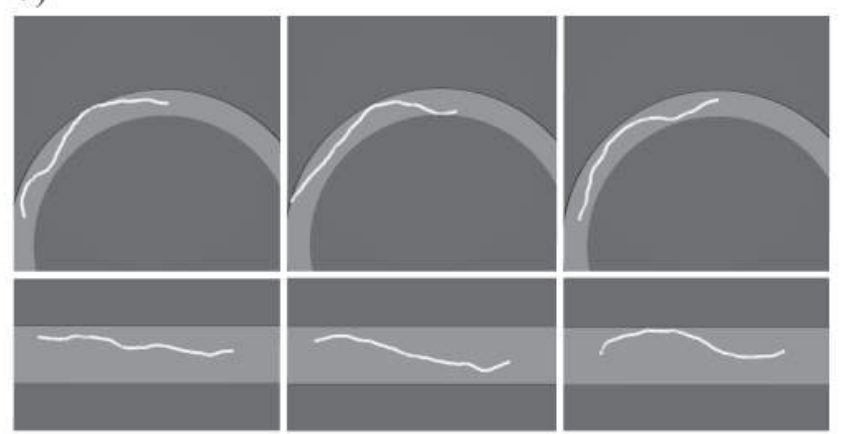

Fig. 2. Snapshots of fluctuating actin filaments. a) Experimental data for straight and curved channels. b) Simulated data for straight and curved channels. Scale bars are $10 \mu \mathrm{m}$.

The scaling is governed by two length scales, the persistence length $L_{P}$ and the deflection length $\lambda$, for which the Odijk scaling relation $\lambda=a L_{P}^{1 / 3} d^{2 / 3}$ holds [17] with a numerical prefactor $a \simeq 0.76$, which we determine by Monte Carlo (MC) simulations.

Our approach can be generalized to curved channels with an arbitrary shape. The results assume a particularly simple form provided that the channel radius of curvature is large compared to the deflection length. The center line of a curved channel with rectangular cross-section represents a contour in the two-dimensional focal plane of the microscope. We parameterize this two-dimensional contour $\mathbf{r}_{0}(s)$ using its arclength $s$. The position of the filament segments can be described by displacements normal to the channel direction. We introduce the unit tangent $\mathbf{t}_{0}(s)=\partial_{s} \mathbf{r}_{0}$ to the channel contour and the outward normal $\mathbf{n}_{0}(s)=-R(s) \partial_{s} \mathbf{t}_{0}$, where $R(s)$ is the local radius of curvature of the channel center line. For a straight channel we have $\mathbf{r}_{0}(s)=s \mathbf{e}_{x}$ and zero channel curvature or infinite radius of curvature. Analogously to the straight channels we describe filament fluctuations within the focal plane by displacements $z(s)$ perpendicular to the local channel direction. The filament's contour in the focal plane is given by

$$
\mathbf{r}(s)=\mathbf{r}_{0}(s)+z(s) \mathbf{n}_{0}(s) .
$$

We assume that the filaments are only weakly bent with small displacement gradients $\partial_{s} z \ll 1$. With the parametrization in equation (3) it follows that the bending energy of the filament is

$$
\mathcal{H}_{b}=\int_{0}^{L} \mathrm{~d} s \frac{\kappa}{2}\left[R^{-2}(s)-R^{-3}(s) z(s)+\left(\partial_{s}^{2} z\right)^{2}\right],
$$

where the first term is the contribution of the background curvature of the channel, that is, the bending energy for a filament lying in the center of the curved channel. The second term represents the contribution of an effective force that pushes the filament outwards. The last term is the contribution of shape fluctuations of the filament within the channel. In the bending energy, we neglect higher order terms in displacement gradients $\partial_{s} z$ as well as terms of the order of $\mathcal{O}\left(R^{-4} z^{2}\right)$ and $\mathcal{O}\left(R^{-2}\left(\partial_{s} z\right)^{2}\right)$. These terms are small compared to the term $\sim\left(\partial_{s}^{2} z\right)^{2}$ if $\lambda \ll R$. The condition $R \gg \lambda$ implies a separation of length scales of filament fluctuations and channel geometry: The typical length scale $\lambda$ for filament fluctuations along the channel needs to be much smaller than the radius of curvature of the channel geometry to apply this description.

Locally, the channel potential acts on the displacement $z(s)$ perpendicular to the channel in the same manner as for a straight channel. Therefore, we proceed as for straight channels and approximate the steric potential for hard channel walls by a harmonic potential, which leads to a Hamiltonian $\mathcal{H}_{R}=\mathcal{H}_{b}+\int_{0}^{L} \mathrm{~d} s \frac{K}{2} z^{2}(s)$.

For large radii of curvature, the tangent correlations of filaments in curved channels are simply given by multiplying the background correlations $\mathbf{t}_{0}(s) \cdot \mathbf{t}_{0}(s+l)$ caused by the channel geometry with the result of a straight channel,

$$
\langle\mathbf{t}(s) \cdot \mathbf{t}(s+l)\rangle=\left(\mathbf{t}_{0}(s) \cdot \mathbf{t}_{0}(s+l)\right)\langle\mathbf{t}(s) \cdot \mathbf{t}(s+l)\rangle_{\text {straight }},
$$

where $\langle\mathbf{t}(s) \cdot \mathbf{t}(s+l)\rangle_{\text {straight }}$ is the tangent correlation function for a straight channel with zero curvature, $R^{-1}=0$. In the experiments presented here we use channels with constant curvature $R$. In this case, equation (5) leads to

$$
\begin{aligned}
& \langle\cos \theta(l)\rangle=\cos \left(\frac{l}{R}\right) \\
& \times\left[1-\frac{\lambda}{2 \sqrt{2} L_{P}}\left(\cos \left(\frac{\pi}{4}\right)-\cos \left(\frac{\pi}{4}+\frac{l}{\lambda}\right) \exp \left(\frac{-l}{\lambda}\right)\right)\right] .
\end{aligned}
$$

In particular, this means that the ratio $\langle\cos \theta(l)\rangle / \cos (l / R)$ assumes the scaling form of equation (1) with the shape function of equation (2).

We use MC simulations to show that equation (6) is still valid if the same definition of the deflection length is used as for straight channels. We perform MC simulations for filament parameters $L=100 \mu \mathrm{m}$ and $L_{P}=15 \mu \mathrm{m}$, and channel width $d=2.5 \mu \mathrm{m}$ with constant radii of curvature $(R=30,50,100,150$, or $200 \mu \mathrm{m})$. The ratio $\langle\cos \theta(l)\rangle / \cos (l / R)$ should exhibit the same scaling properties as for a straight channel. We use this ratio in the regime $l / R \ll \pi / 2$, where the cosine is larger than zero, to perform the same rescaling as for straight channels. If the condition $R \gg \lambda$ is fulfilled, the length scale $\lambda$ on which the filaments perform unconfined fluctuations and 
the length scale $R$ of the curved confining channel decouple. In this case the data can be collapsed onto the master curve

$$
\left(1-\frac{\langle\cos \theta(l)\rangle}{\cos (l / R)}\right) d^{-2 / 3} L_{P}^{2 / 3}=\mathcal{C}_{a}\left(l d^{-2 / 3} L_{P}^{-1 / 3}\right)
$$

with the same scaling function $\mathcal{C}_{a}(x)=a \mathcal{C}(x / a)$ and the same numerical prefactor $a \simeq 0.76$ as for straight channels [14] (see Fig. 5b).

\subsection{Segment distribution}

A confining potential also influences the temporal and spatial distribution of segments of the filament within the conformational space. Therefore, the channel geometry gives rise to a characteristic distribution of filament segments in the $z$-direction, which can be measured in the present experiments. In the following, we consider a typical center segment, which is at least a distance $\lambda$ away from the filament ends. Segments at the filament ends exhibit a slightly different scaling behavior. In contrast to flexible macromolecules, each segment of a semiflexible macromolecule has a well-defined tangent vector, and we have to consider the compound probability density $P(z, v)$ for segment displacements $z$ and displacement gradients $v \equiv \partial_{s} z \cdot P(z, v) \delta z \delta v$ is the probability to find a center segment within the range $[z, z+\delta z]$ and with a displacement gradient in the interval $[v, v+\delta v]$. This probability density obeys a scaling form, which is governed by two exponents [23-26]: One exponent $\theta_{r}$ describes the depletion of segments in front of the confining wall, and the other exponent $\chi_{r}$ characterizes the probability of contacts with the wall. The detailed scaling form is given in the appendix.

The corresponding value $P(z, 0)$ is the contact probability, for which we find

$$
P(z, 0) \approx z^{\theta_{r}} d^{-4 / 3-\theta_{r}} L_{P}^{1 / 3} .
$$

The contact exponent $\chi_{r}$ is defined such that it describes the scaling of this contact probability with the correlation length along the polymer, which is given by the deflection length, $P(z, 0) \sim \lambda^{-\chi_{r}}[26]$. Using the scaling law $d \sim \lambda^{3 / 2}[17]$ together with equation $(8)$ this leads to an exponent relation [26]

$$
\chi_{r}=2+3 \theta_{r} / 2 .
$$

This shows that the depletion exponent $\theta_{r}$ and the contact exponent $\chi_{r}$ are not independent and it is sufficient to determine one of these exponents to completely describe the scaling behavior of the segment distribution. The exponents are characteristic properties of a semiflexible polymer at a hard wall, which have been calculated analytically using transfer matrix techniques [24,25]:

$$
\theta_{r}=1 / 3 \quad \text { and } \quad \chi_{r}=5 / 2 .
$$

In the experiments, the $v$-integrated segment distribution is measured, that is, the distribution of segment positions irrespective of their orientation. The corresponding contact probability of finding a segment close to the wall with arbitrary tangent $v$ is

$$
\begin{aligned}
P(z) & \sim z^{1 / 3+\theta_{r}} d^{-2 \chi_{r} / 3} \\
& \sim z^{2 / 3} d^{-5 / 3}
\end{aligned}
$$

Thus, the probability $P(z) \delta z$ to find a segment of a filament in a range $[z, z+\delta z]$ in close proximity to a channel wall scales with $z^{1 / 3+\theta_{r}}$ as a function of $z$ at fixed $d$, and with $d^{-2 \chi_{r} / 3} \sim \lambda^{-\chi_{r}}$ as a function of $d$ at a fixed distance $z$.

The scaling behavior of $P(z)$ given in equation (11) can be specified in terms of a shape function $\tilde{\Omega}(y)$. MC simulations can be used to determine $\tilde{\Omega}(y)$ and to verify the scaling behavior. To compare this to experimental data, we perform MC simulations of long filaments $(L=35 \mu \mathrm{m})$ with $L_{P}=13 \mu \mathrm{m}$ in channels with different widths $d=1.5,4.2,5.8$, and $9.8 \mu \mathrm{m}$. These values correspond to the experimental situation. The distribution $P(z)$ is averaged over all segments along the filament to improve the statistics. For long filaments, this does not affect our results regarding the scaling properties of the distribution.

To determine the shape function $\tilde{\Omega}(y)$, we rescale the simulation data. We bin the segment distribution data into $n_{\text {bin }}=100$ bins of length $\delta z=d / n_{\text {bin }}$ and show the probabilities $P(z) d / n_{\text {bin }}$ as a function of the rescaled coordinate $z / d$. We should find a collapse of all data to a curve

$$
P(z) \frac{d}{n_{\text {bin }}}=\mathcal{P}\left(\frac{z}{d}\right)
$$

with a scaling function

$$
\mathcal{P}(y)=n_{\text {bin }}{ }^{-1}[y(1-y)]^{2 / 3} \tilde{\Omega}(y)
$$

for a depletion exponent $\theta_{r}=1 / 3$.

In order to test whether we can fit the data satisfyingly well using a constant value $\tilde{\Omega}(y)=\tilde{\Omega}$ for the shape function, we use the scaling function

$$
\mathcal{P}(y)=3.41 n_{\text {bin }^{-1}}[y(1-y)]^{2 / 3},
$$

corresponding to a constant

$$
\tilde{\Omega}(y)=\tilde{\Omega}=\frac{7 \Gamma(1 / 3)}{2^{2 / 3} \sqrt{3 \pi} \Gamma(5 / 6)} \simeq 3.41,
$$

which is determined by the normalization condition $\int_{0}^{d} \mathrm{~d} z P(z)=1$ or $\tilde{\Omega}^{-1}=\int_{0}^{1} \mathrm{~d} y[y(1-y)]^{2 / 3}$. In equation (15), $\Gamma(x)$ is the Gamma function [27].

\section{Results and discussion}

\subsection{Segment distribution in microchannels}

Previously, we have reported careful analysis of tangent correlation data derived from experiments with long 
( $L \geq 30 \mu \mathrm{m})$ semiflexible filaments in straight confining microchannels $[13,14]$. These studies reveal a unique behavior of tangent correlations. Briefly, tangent correlations of confined filaments deviate from the known exponential decay of the tangent correlations of freely fluctuating filaments $[28,29]$. The overall correlation increases as the channel width decreases, as does the saturation level for very large distances $l$ between two macromolecule segments along the filaments' contour. A pronounced minimum evolves and is shifted towards larger values of $l$ as the channel width increases. The qualitative picture that emerges from the results is as follows: At distances $l \sim \lambda$ the confining potential induces turns of the filament's contour leading to the minimum in the tangent correlation function. On length scales $l<\lambda$, filament segments are approximately free and the correlation function resembles the exponentially decaying free correlation function in this regime. On length scales $l \gg \lambda$, the confining potential leads to an alignment of the filament and therefore, to a characteristic plateau in the tangent correlations. Such a plateau cannot be explained by a stiffening of the filaments as it can be observed for filaments with larger persistence length but in contrast is a unique feature of the confinement effect. The value of the plateau in the tangent correlations is consistent with a picture, where the filament is viewed as an ensemble of roughly uncorrelated segments of length $\lambda$.

The presence of channel walls does not only influence the local tangential orientation of confined filaments. The distribution of filament segments with respect to the position within the channels also changes distinctly once wall potentials are introduced to the system. Analyzing the segment distribution of filaments in proximity of the channel walls sheds light on certain scaling laws that characterize the segment depletion in front of the walls. In Section 3.2, we introduced two exponents which are characteristic of depletion effects of semiflexible macromolecules: $\chi_{r}$ for the contact probability to the wall along the polymer, that is to say, parallel to the wall, and $\theta_{r}$ for the segment distribution perpendicular to the wall [26].

In our experiments we are able to measure these exponents for single macromolecules. The segment distributions $P(z)$ for different channel widths $(d=1.5,4.2,5.8$, and $9.8 \mu \mathrm{m})$ are shown in a double logarithmic plot against $z$ in Figure 3a.

To improve the statistics, the data for $P(z)$ are averaged over the whole length; since the filaments are sufficiently long ( $L \geq 30 \mu \mathrm{m})$, our results are not affected. In addition, we make use of the symmetry $P(z)=P(d-z)$ and also average over contours mirrored at the center line of the channel $(z=d / 2$, since we choose $z=0$ and $z=d$ as the positions of the channel walls).

The solid line in Figure 3a corresponds to the scaling law $P(z) \propto z^{2 / 3}$ that we obtain in equation (11) for the probability to find a segment of the filament at a position $z$ close to the wall within the channel. The improved statistics of the data for smaller $d$ are obvious. Although all four data sets show the scaling, a smoother course is observed for smaller channels. This is due to the smaller conforma- tional space in the case of smaller channels, generally leading to better statistics. The observed scaling $P(z) \propto z^{2 / 3}$ is typical of semiflexible filaments and gives direct experimental evidence for a scaling exponent $\theta_{r}=1 / 3$, as it has been obtained analytically $[24,25]$. This exponent is characteristic of a semiflexible chain governed by its bending energy. For a flexible Gaussian chain, in contrast, one would expect an exponent $\theta_{r}=2$ and $P(z) \propto z^{\theta_{r}}$ close to a wall $[30,31]$. The slope with which the segment distribution increases in close proximity to the channel walls describes the depletion of segments near the wall owing to deflection of the filament by the potential. The small exponent $\theta_{r}=1 / 3$ for semiflexible polymers indicates that the influence of the confining wall potential on the filaments is extended quite far into the channel-mediated by the intrinsic semiflexible properties of the filament. Generally, we expect an exponent $\theta_{r}<0$ for attractive interactions, $\theta_{r}=0$ in the absence of interactions, and $\theta_{r}>0$ for repulsive interactions, which give rise to depletion as presented here. Thus, the result $\theta_{r}=1 / 3>0$ also demonstrates that there is no residual unspecific attraction between filaments and channel walls in our experiment.

The second exponent to be considered, $\chi_{r}$, describes the scaling of $P(z)$ with the channel width $d$ for a fixed $z$ close to the wall (see Eq. (11)). Analysis of the values for $P(z)$ requires careful renormalization of the data. Subsequently, we average the frequency values of the first nine data points $\left(z \leq z^{*} \approx 0.6 \mu \mathrm{m}\right)$ and plot the results against $d$ (see Fig. $3 \mathrm{~b}$ ). The value $z^{*} \approx 0.6 \mu \mathrm{m}$ is chosen since it represents about half the width of the smallest channel and can thus be applied to all data sets discussed here. According to the relation equation (11), we expect a scaling $P\left(z^{*}\right) \propto d^{-2 \chi_{r} / 3}$. The dotted line in Figure $3 \mathrm{~b}$ corresponds to the scaling law $P\left(z^{*}\right) \propto d^{-5 / 3}$. The experiment thus supports an exponent value $\chi_{r}=5 / 2$, as it has been obtained analytically [24,25].

The value $\chi_{r}=5 / 2$ also has important consequences for the proper interpretation of the deflection length $\lambda$ in terms of collisions with the channel walls. It is often stated that the deflection length $\lambda$ is the "average distance between collisions" with the confining walls (see for example Ref. [17]). The result $\chi_{r}=5 / 2>1$ shows that such a statement is not correct. Since the contact probability is proportional to $\lambda^{-\chi_{r}}$, actual contacts with the wall are much rarer than one collision per deflection length if $\chi_{r}>1$. An analogous finding has been pointed out in reference [32] in the context of fluid two-dimensional membranes. The deflection length $\lambda$ is the correlation length of the segment distribution. As such, it does not give the average distance between collisions but actually characterizes the exponentially decaying tails of the distribution of contour lengths between contacts: The probability of finding a long polymer segment $L \gg \lambda$ between two contacts decays as $\sim \exp (-L / \lambda)$.

Both scaling parameters, $\theta_{r}$ and $\chi_{r}$, describe the experimental results on a length scale of $\leq 1 \mu \mathrm{m}$ in the proximity of the channel walls. By extracting the contour line from the micrographs and analyzing the statistical probability to find a filament segment at a specific distance 
a)

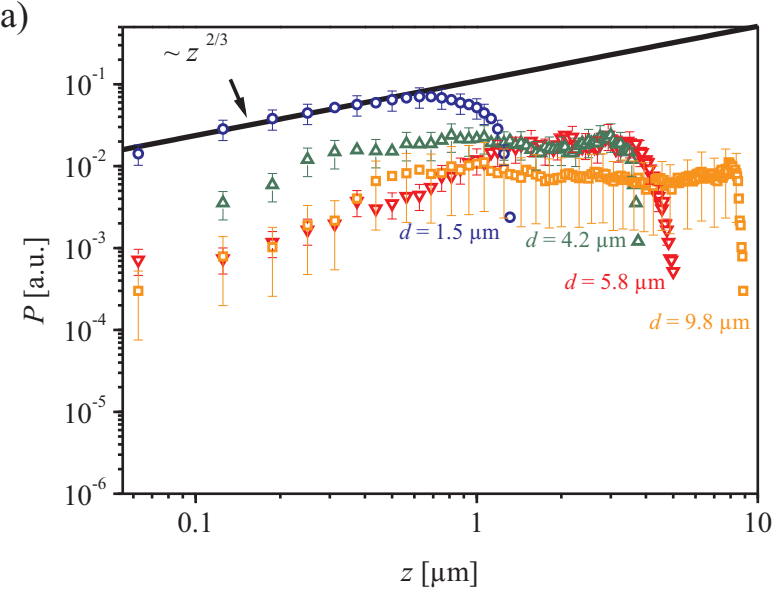

c)

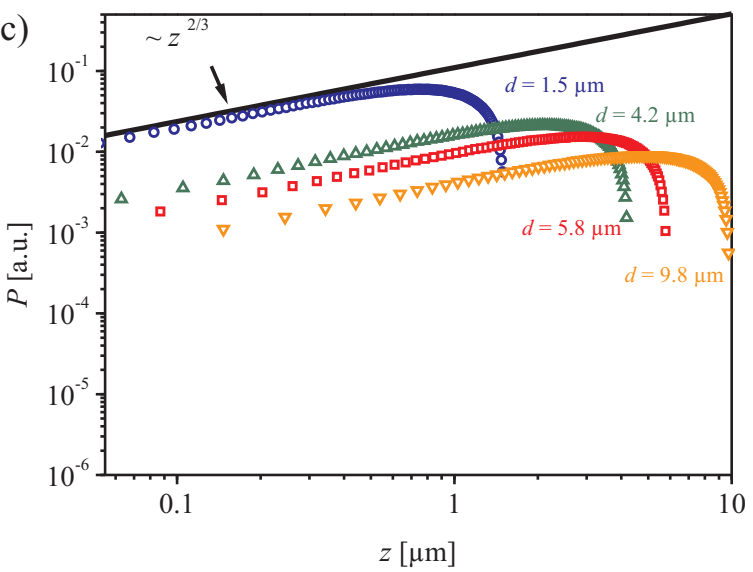

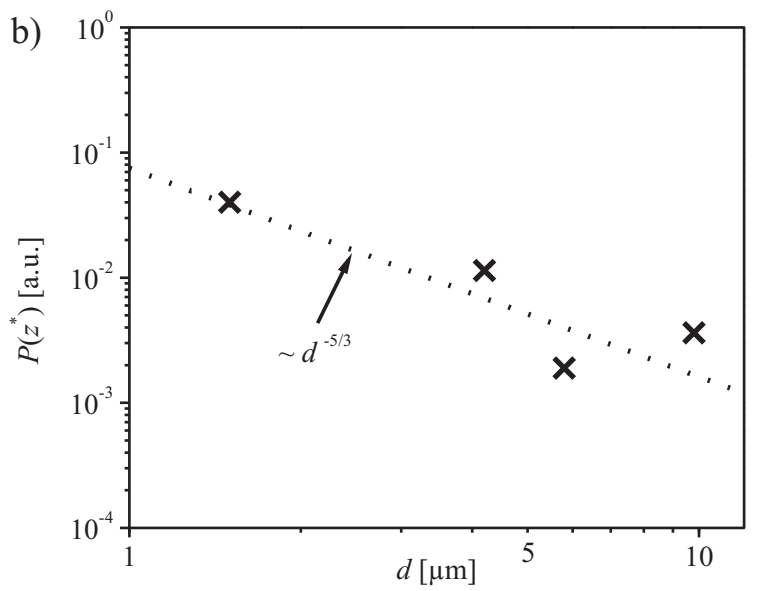

d)

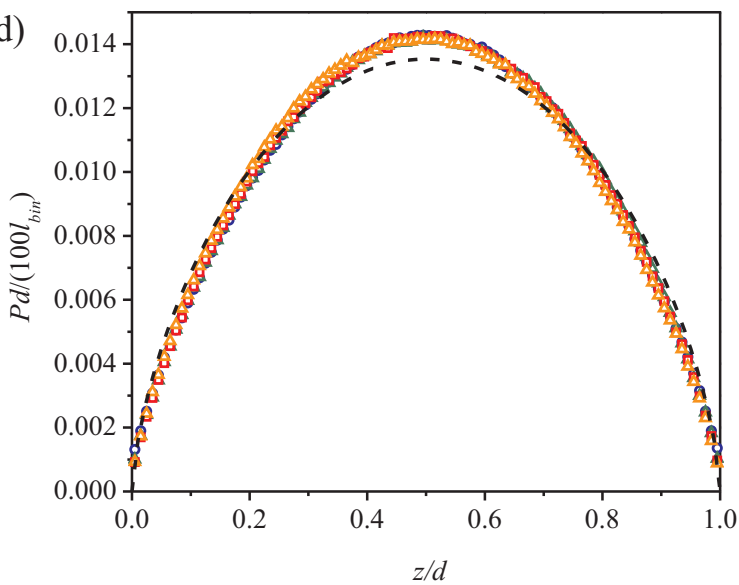

Fig. 3. a) Double-logarithmic plot of the experimental segment distributions which scale as $z^{2 / 3}$. b) Contact probability to the wall which scales as $d^{-5 / 3}$. c) Double-logarithmic plot of the simulated segment distributions which scale as $z^{2 / 3}$ as well. d) Simulation data as shown in c collapsed onto a single master curve (dashed line).

from the wall, we can make educated assumptions about the behavior of the filaments on a length scale smaller than the optical resolution.

In this section, we present experimental evidence for the two exponent values $\theta_{r}=1 / 3$ and $\chi_{r}=5 / 2$. Previously we have confirmed the scaling law $\lambda \propto d^{2 / 3}$ experimentally [14]. This relation provides a direct measurement of the roughness exponent $\zeta=3 / 2$, hearkening back to the condition $\left\langle z^{2}\right\rangle \sim d^{2}$ with $\left\langle z^{2}\right\rangle \propto \lambda^{2 \zeta}$ for a filament segment of length $\lambda$. Determining all three exponents $\zeta, \theta_{r}$, and $\chi_{r}$ experimentally, we achieve a complete characterization of fluctuations of semiflexible polymers confined by hard walls. We further confirm our findings by providing extensive MC simulations showing the same scaling behavior.

Figure 3c shows simulated data for the same parameters as the experimental data in Figure 3a (channels widths, persistence length, filament length). Note, though, that infinitely thin filaments are assumed in these simulations. The simulations and the experimental data display a striking similarity, both qualitatively and quantitatively. Figure 3d shows the same simulation data again, but rescaled onto a single master curve according to equation (12) (dashed line). All data sets collapse nicely onto the scaling function and thus provide strong support of our experimental findings.

\subsection{Curved channels}

In biological systems, for example the cytoskeleton or the densely packed DNA in cell nuclei, as well as in microfluidic applications, the confining geometry is usually much more complex than a simple straight channel. Therefore, we add more complexity and present experiments and simulations where we confine actin filaments in channels with varying radii of curvature $R$. We thus investigate the influence of additional bending on confined filaments. $R$ is defined as the radius of the center line of the channel, in other words the mean value of the inner radius $R_{i}$ and the outer radius $R_{0}$ (see Fig. $1 \mathrm{~b}$ and c). In Figure $4 \mathrm{a}$ the tangent correlation functions for $d=2.5 \mu \mathrm{m}$ wide channels and various values of $R$ are shown. Each curve represents data from an individual filament and the data are averaged over all recorded conformations and the whole length of the filament. All filaments have a similar contour length $L \approx 20 \mu \mathrm{m}$. The radii of curvature lie between $13.5 \mu \mathrm{m}$ and $143.5 \mu \mathrm{m}$. These values are on the same or- 
a)

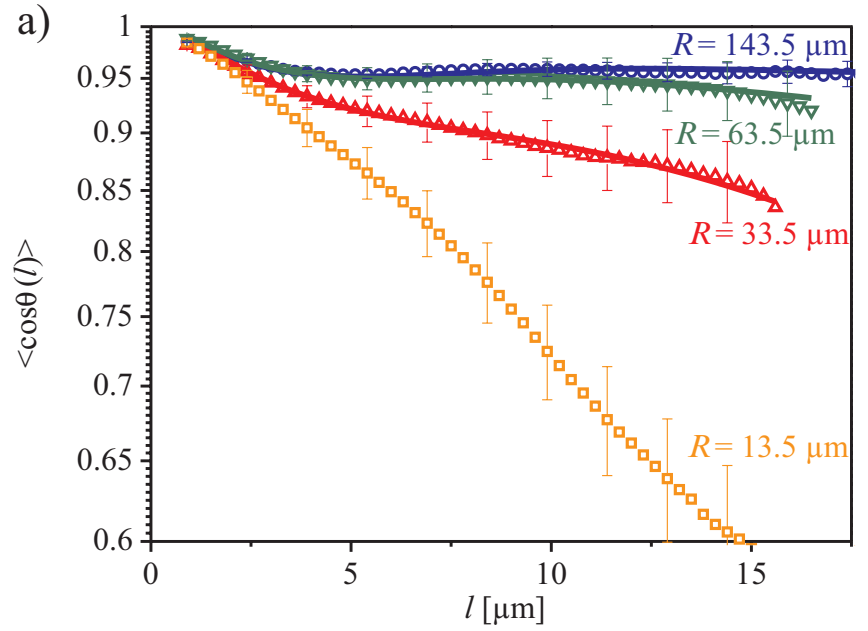

b)

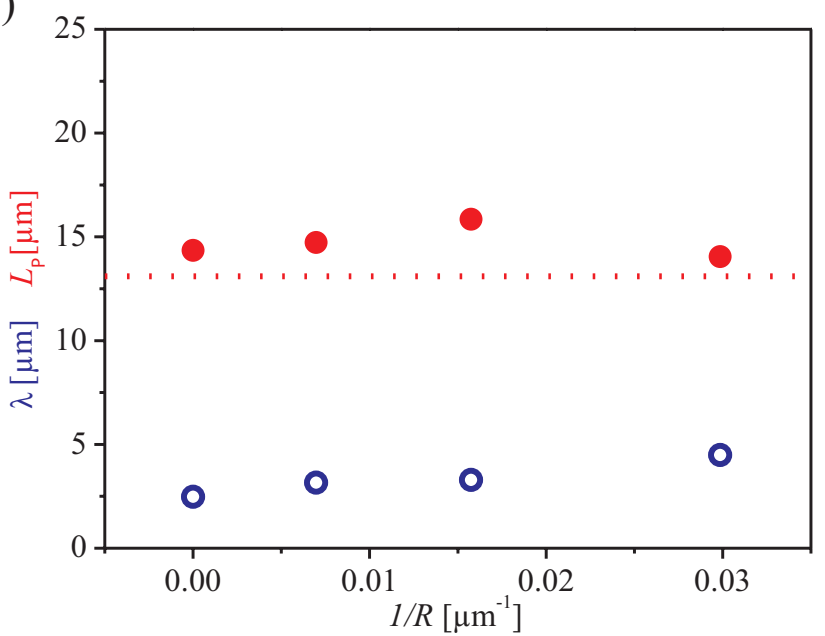

Fig. 4. a) Tangent correlations of actin filaments in curved channels (open symbols) with fits (solid lines); $d=2.5 \mu \mathrm{m}$, different radii of curvature $R$. b) Fit parameters $\lambda$ and $L_{P}$. For comparison $\lambda$ and $L_{P}$ of straight channels with $d=1.5 \mu \mathrm{m}$ $(1 / R=0)$ are plotted as well. Dotted line: mean value for $L_{P}$ in straight channels.

der of magnitude as the filament length $L$, the persistence length $L_{P}$, the channel width $d$, and the deflection length $\lambda$. Therefore, we expect an influence of the radius of curvature on the filaments' behavior. More specifically, the radii of curvature range from $R \sim L_{P}$ to $R \gg L_{P}$ thus comprising the tightly as well as the weakly bent regime [19]. Compared to straight channels, the fluctuations in curved channels appear clearly less correlated. This effect is more pronounced in channels with smaller $R$ although in all cases the filaments are yet more correlated than unconfined filaments.

To quantify these results, we fit the data with equation (6), which applies if the radius of curvature is larger than the deflection length, or $R \gg \lambda$ such that the background curvature introduced by the channel is small compared to the typical filament curvature $1 / \lambda$ from thermal fluctuations. We take into consideration a Gaussian correction factor accounting for the fact that microscopy images generally show finite width contour lines of the

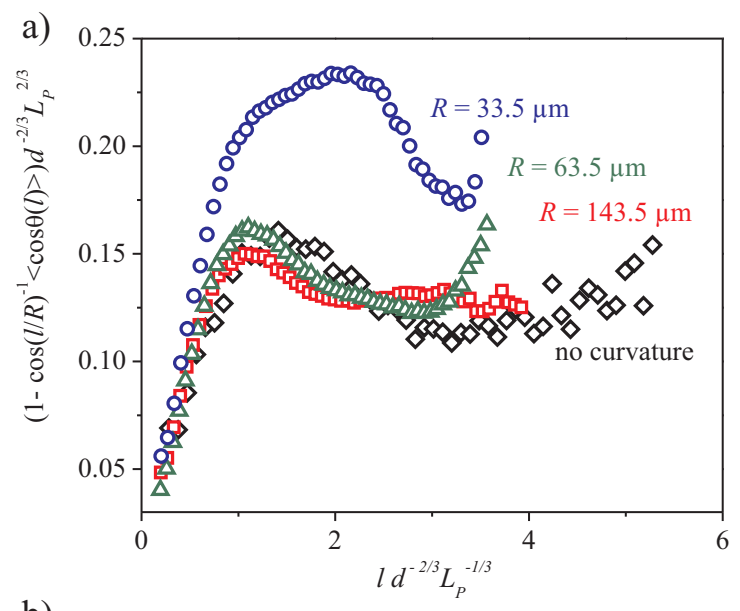

b)

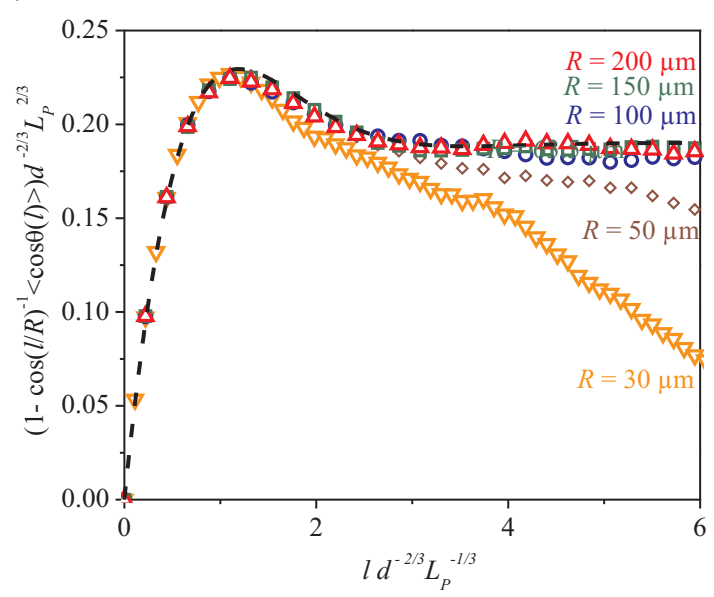

Fig. 5. Tangent correlations of filaments in curved channels, collapsed onto a master curve. a) Experimental data as shown in Figure 4a. b) Simulation data.

filaments [14]. The radius of curvature of the channels is measured and introduced into the equation and is not used as a free fit parameter. The fitting functions are shown as solid lines in Figure 4a. For $R=13.5 \mu \mathrm{m}$, the notion of the background correlation as described in Section 3 breaks down and thus we are not able to obtain values for the fit parameters.

In Figure $4 \mathrm{~b}$ both fit parameters, $\lambda$ and $L_{P}$, are plotted against the curvature, $1 / R$. All values for $L_{P}$ lie in the expected range, close to the mean value for straight channels, $L_{P}=13.1 \mu \mathrm{m}$ (dotted line in Fig. 4b). The differences between individual filaments are similar to those observed for straight channels [14]. The deflection length $\lambda$ as well is almost constant for the larger radii $R$ and slightly larger than the value which we obtain for straight channels with $d=1.5 \mu \mathrm{m}$ (value for $1 / R=0$, plotted for comparison). This is to be expected for the weakly bent regime, where the fluctuations of the filaments are not influenced very much by the bending [19]. Thus the fluctuations can be considered as superimposed to a background correlation and equation (6) can be applied to fit the data. The situation is different for tightly bent filaments, that is, small radii of curvature. While for very small values 
of $R$ we cannot apply the background correlation of the channels to the filaments' fluctuations, in the case of an intermediate bending regime for the channels, we observe an increase of $\lambda(R=33.5 \mu \mathrm{m})$. If a filament is tightly bent, long wavelength fluctuations are frozen out, at the same time resulting in smaller fluctuation amplitudes [19]. If a channel wall is involved we do not observe an effect on $\lambda$ as long as the fluctuation amplitudes are still larger than the channel width. However, once the bending becomes strong enough such that the amplitudes become smaller than the channel width, the original notion of the deflection length is no longer valid. Thus, we cannot superimpose the "unbent" fluctuations by a background correlation.

We can rescale the data for filaments in curved channels as shown in Figure 4a to collapse the data on a single curve. This is done in two steps: by i) multiplying the data by the prefactor in equation (6) that accounts for the background curvature and ii) rescaling the data according to the Odijk scaling. The resulting data plots are shown in Figure 5a. The data for the strongest curvature, $R=13.5 \mu \mathrm{m}$, cannot be rescaled since values for $\lambda$ and $L_{P}$ could not be obtained. In comparison to these experimental results, Figure 5b shows simulation data for tangent correlations of filaments in curved channels, also collapsed onto a single master curve. In both the experiment and the simulation we observe, that only data sets for sufficiently large radii of curvature $(R \geq 63.5 \mu \mathrm{m}$ and $R \geq 100 \mu \mathrm{m}$, respectively) can be collapsed onto a master curve with data for straight channels. However, the data for smaller $R$ show a very different behavior. In the case of small radii of curvature the notion of a superposition of the tangent correlations of the filament and the background correlation breaks down. At the same time the simulations come to a limit for these parameters. Nevertheless, these results show that for strongly bent channels the background tangent correlation and the tangent correlation of the filament fluctuations cannot be superimposed as for more weakly bent channels.

\section{Conclusions}

We present a consistent description of semiflexible filaments in confining geometries. We combine singlemolecule experiments with Monte Carlo simulations and analytical descriptions to characterize the macromolecules' behavior in terms of tangent correlations and segment distributions, taking into account channel width and curvature. We are able to directly measure three scaling exponents which are characteristic for semiflexible filaments, in particular we measure the depletion exponent $\theta_{r}=1 / 3$, and the contact exponent $\chi_{r}=5 / 2$, which characterize the scaling properties of the segment distribution. We thus show that confined actin can indeed be described in terms of the worm-like chain model on all length scales accessible to optical microscopy. At the same time, we experimentally prove the general scaling laws for semiflexible-polymer physics. Furthermore, we demonstrate that for weakly bent channels the background curvature leads to a background tangent correla- tion onto which the tangent correlation of the shape fluctuations of the filament is superimposed. For strongly bent channels this notion breaks down due to a freezing-out of long-wavelength fluctuations. These results represent a first step towards the characterization of single-filament behaviour in complex confinement geometries such as in reconstituted-biopolymer networks or in in vivo networks in cells.

We gratefully acknowledge fruitful discussions with Stephan Herminghaus, Reinhard Lipowsky, Holger Stark, and Heather Evans. This project was supported by the DFG in the framework of the Emmy Noether Program (PF 375/2) and of the Priority Program SPP 1164 "Nano- and Microfluidics" (PF $375 / 4)$.

\section{Appendix A. Tangent correlations in curved channels}

In the following section we discuss the behavior of semiflexible filaments in curved channels. As for a straight channel it is sufficient to consider the projection of the filament contour into the two-dimensional focal plane, the $x z$ plane. Fluctuations in the perpendicular $y$-direction decouple for a weakly bent filament. In this two-dimensional plane, we consider a curved channel whose centerline is given by the contour $\mathbf{r}_{0}(s)$, where $s$ is the arclength of the channel contour. We introduce the unit tangent vector $\mathbf{t}_{0}(s)=\partial_{s} \mathbf{r}_{0}$ to the channel contour and the unit outward normal vector $\mathbf{n}_{0}(s)=-R(s) \partial_{s} \mathbf{t}_{0}(s)$, where $R(s)$ is the local radius of curvature of the channel center line, that is, $R^{-1}(s)$ is the local channel curvature. We also find $\partial_{s} \mathbf{n}_{0}(s)=R^{-1}(s) \mathbf{t}_{0}(s)$. We describe the filament's contour within the focal plane by displacements $z(s)$ perpendicular to the local channel direction,

$$
\mathbf{r}(s)=\mathbf{r}_{0}(s)+z(s) \mathbf{n}_{0}(s),
$$

and assume that filaments are only weakly bent with small displacement gradients $\partial_{s} z \ll 1$. The (unnormalized) tangent vectors of the filament contour (Eq. (A.1)) are

$$
\mathbf{t}(s)=\mathbf{t}_{0}(s)\left(1+z(s) / R(s)-\frac{1}{2}\left(\partial_{s} z\right)^{2}\right)+\mathbf{n}_{0}(s)\left(\partial_{s} z\right),
$$

where we neglect terms of order $\mathcal{O}\left(z^{2} R^{-1}\right)$ and $\mathcal{O}\left(z\left(\partial_{s} z\right) R^{-1}\right)$. The arclength element $\mathrm{d} s\left|\partial_{s} \mathbf{r}\right|$ of the filament's contour (Eq. (A.1)) is given by

$$
\mathrm{d} s\left|\partial_{s} \mathbf{r}\right| \approx \mathrm{d} s\left(1+z(s) / R(s)-\frac{1}{2}\left(\partial_{s} z\right)^{2}\right) .
$$

For a contour given by equation (A.1) the filament's bending energy becomes

$$
\mathcal{H}_{b}=\int_{0}^{L} \mathrm{~d} s \frac{\kappa}{2}\left[R^{-2}(s)-R^{-3}(s) z(s)+\left(\partial_{s}^{2} z\right)^{2}\right] .
$$


In equations (A.3) and (A.4), we neglect some terms of the order of $\mathcal{O}\left(R_{0}^{-4} z^{2}\right)$ and $\mathcal{O}\left(R_{0}^{-2}\left(\partial_{s} z\right)^{2}\right)$ in the integrand. To analyze the validity of these approximations, we consider an almost straight channel. It can be shown that fluctuations of displacement derivatives scale as $\left\langle\left(\partial_{s}^{n} z\right)^{2}\right\rangle_{\text {straight }} \sim$ $\lambda^{3-2 n} / L_{P}$ for straight channels. Therefore, all neglected terms in equation (A.3) are small compared to the term $\sim\left(\partial_{s} z\right)^{2}$ and all neglected terms in equation (A.4) are small compared to the term $\sim\left(\partial_{s}^{2} z\right)^{2}$ as long as $\lambda \ll R$. Equations (A.2), (A.3), and (A.4), represent the leadingorder terms of an expansion in $\lambda / R$.

Filament fluctuations are governed by the sum of the bending energy and a confining potential, which acts on the displacement $z(s)$ perpendicular to the channel. In the same manner as for straight channels, we replace the steric potential $V_{d}(z)$ for channel walls by a parabolic potential $K z^{2} / 2$, which leads to the Hamiltonian

$$
\begin{aligned}
\mathcal{H}_{R}=\int_{0}^{L} \mathrm{~d} s[ & \frac{\kappa}{2}\left(R^{-2}(s)-R^{-3}(s) z(s)+\left(\partial_{s}^{2} z\right)^{2}\right) \\
& \left.+\frac{K}{2} z^{2}(s)\right]
\end{aligned}
$$

for filament fluctuations in a curved channel. Taking into consideration equation (A.2), the tangent correlation function becomes

$$
\begin{aligned}
& \langle\mathbf{t}(s) \cdot \mathbf{t}(s+l)\rangle=\left(\mathbf{t}_{0}(s) \cdot \mathbf{t}_{0}(s+l)\right) \\
& \quad \times\left[1+\frac{\langle z(s)\rangle}{R(s)}+\frac{\langle z(s+l)\rangle}{R(s+l)}-\frac{1}{2}\left\langle\left(\partial_{s} z(s)-\partial_{s} z(s+l)\right)^{2}\right\rangle\right] \\
& \quad+\left(\mathbf{t}_{0}(s) \cdot \mathbf{n}_{0}(s+l)\right)\left\langle\partial_{s} z(s+l)-\partial_{s} z(s)\right\rangle .
\end{aligned}
$$

From the quadratic Hamiltonian equation (A.5) we find for the shift of the mean filament contour with respect to the center line of the channel by balancing the force term (second summand in Eq. (A.5)) and the potential term (fourth summand):

$$
\langle z(s)\rangle / R(s)=\frac{\kappa}{K R^{4}(s)}=\frac{\lambda^{4}}{4 R^{4}(s)}
$$

and

$$
\left\langle\partial_{s} z(s)\right\rangle=-\frac{3 \lambda^{4}}{4 R^{4}(s)} \partial_{s} R(s) .
$$

In the limit $\lambda \ll R$, expression (A.8) becomes very small and for a channel of constant curvature $R$, as used in the experiments, it vanishes. Because the Hamiltonian in equation (A.5) is quadratic, the correlation function $\left\langle\left(\partial_{s} z(s)-\partial_{s} z(s+l)\right)^{2}\right\rangle$ is essentially given by the same result as for a straight channel:

$$
\begin{aligned}
\left\langle\left[\partial_{s} z(s)-\partial_{s} z(s+l)\right]^{2}\right\rangle & =\left\langle\left[\partial_{s} z(s)-\partial_{s} z(s+l)\right]^{2}\right\rangle_{\text {straight }} \\
& +\left[\left\langle\partial_{s} z(s)-\partial_{s} z(s+l)\right\rangle\right]^{2}
\end{aligned}
$$

From equation (A.8) and $\left\langle\left(\partial_{s} z\right)^{2}\right\rangle_{\text {straight }} \sim \lambda / L_{P}$ it follows that the second contribution in (A.9) can be neglected for $\lambda \ll R\left(R\left(\partial_{s} R\right)^{2} / L_{P}\right)^{1 / 7}$. Likewise, the contributions linear in $\langle z\rangle$ in equation (A.6) can be neglected for $\lambda \ll$ $R\left(R / L_{P}\right)^{1 / 3}$ and $\lambda \ll R\left(R \partial_{s} R / L_{P}\right)^{1 / 3}$. These conditions are essentially fulfilled for radii of curvature much larger than the deflection length, $R \gg \lambda$. In this limit and in the approximation of a weakly bent filament, the arclength $s$ of the channel is also essentially identical to the arclength of the filament (see Eq. (A.3)). In the limit $R \gg \lambda$, we finally obtain

$$
\begin{aligned}
& \langle\mathbf{t}(s) \cdot \mathbf{t}(s+l)\rangle \approx\left(\mathbf{t}_{0}(s) \cdot \mathbf{t}_{0}(s+l)\right)\langle\mathbf{t}(s) \cdot \mathbf{t}(s+l)\rangle_{\text {straight }} \\
& =\left(\mathbf{t}_{0}(s) \cdot \mathbf{t}_{0}(s+l)\right)\left[1-\frac{\lambda}{2 \sqrt{2} L_{P}}\right. \\
& \left.\times\left(\cos \left(\frac{\pi}{4}\right)-\cos \left(\frac{\pi}{4}+\frac{l}{\lambda}\right) \exp \left(\frac{-l}{\lambda}\right)\right)\right],
\end{aligned}
$$

where $\langle\mathbf{t}(s) \cdot \mathbf{t}(s+l)\rangle_{\text {straight }}$ is the tangent correlation function for a straight channel with zero curvature, $R^{-1}=0$.

The detailed conditions $\lambda \ll R\left(R / L_{P}\right)^{1 / 3}$ and $\lambda \ll$ $R\left(R \partial_{s} R / L_{P}\right)^{1 / 3}$, under which the tangent correlations factorize as in equation (A.10), are similar to a condition $\lambda \ll R^{2} / L_{P}$ given by Odijk for the regime, where contour fluctuations decouple from background deformations [19]. The criteria are slightly different because Odijk's estimate is based on the scaling behavior of positional fluctuations, whereas we consider tangent fluctuations.

\section{Appendix B. Segment distribution in channels}

In this section we provide more details of the calculation of segment distributions in channels. To derive the scaling form for the segment distribution $P(z, v)$ and the exponents $\theta_{r}$ and $\chi_{r}$ we first note that displacement fluctuations scale as $\left\langle z^{2}\right\rangle \sim \lambda^{3} / L_{P} \sim d^{2}$ and fluctuations of displacement gradients scale as $\left\langle\left(\partial_{s} z\right)^{2}\right\rangle \sim \lambda / L_{P} \sim$ $\left(\left\langle z^{2}\right\rangle / L_{P}^{2}\right)^{1 / 3}$, as follows from equation (1) for large $l \gg \lambda$. Therefore, the center segment distribution of a filament confined in a channel in front of a hard wall at $z=0$ will depend on the scaling variables $z / d$ and $v\left(L_{P} / z\right)^{1 / 3}$. A channel has two walls. We choose $z=0$ and $z=d$ as positions of the channel walls. Then the segment distribution has to obey the symmetry $P(z, v)=P(d-z,-v)$ and a boundary condition $P(0, v)=0$ for all $v \neq 0$. For two walls, the corresponding symmetric scaling variables are $z / d$ and $v\left(L_{P} d / z(d-z)\right)^{1 / 3}$. We obtain a segment distribution of the form

$$
\begin{aligned}
P(z, v)= & d^{-4 / 3} L_{P}^{1 / 3}\left[z(d-z) / d^{2}\right]^{\theta_{r}} \\
& \times \Omega\left(z / d, v\left[L_{P} d / z(d-z)\right]^{1 / 3}\right),
\end{aligned}
$$

where $\Omega(y, u)$ is a shape function defined for $0<y<1$ with the following properties: i) the symmetry $\Omega(y, u)=$ $\Omega(1-y,-u)$; ii) a finite value at $y=u=0$; iii) exponential decay for large $u$; iv) prefactors in equation (B.1) are chosen such that the parameter-free normalization $\int_{0}^{1} \mathrm{~d} y \int_{-\infty}^{\infty} \mathrm{d} u[y(1-y)]^{\theta_{r}+1 / 3} \Omega(y, u)=1$ for the shape 
function gives the usual normalization of the segment distribution, $\int_{0}^{d} \mathrm{~d} z \int_{-\infty}^{\infty} \mathrm{d} v P(z, v)=1$.

The characteristic exponent $\theta_{r}$ describes the segment depletion in front of the channel walls. Close to the wall at $z \approx 0$, we can only find parallel segments with $v=$ 0 (for $v \neq 0$, the scaling function in Eq. (B.1) becomes exponentially small for $z \approx 0$ because the second argument of the scaling function $u \approx v\left(L_{P} / z\right)^{1 / 3}$ becomes large). The corresponding value $P(z, 0)$ is the contact probability, for which we find

$$
P(z, 0) \approx z^{\theta_{r}} d^{-4 / 3-\theta_{r}} L_{P}^{1 / 3} \Omega(0,0) .
$$

The contact exponent $\chi_{r}$ is defined such that it describes the scaling of this contact probability with the correlation length along the polymer, which is given by the deflection length, $P(z, 0) \sim \lambda^{-\chi_{r}}$ [26]. Using the scaling law $d \sim$ $\lambda^{3 / 2}$ [17] together with equation (B.2) this leads to an exponent relation [26]

$$
\chi_{r}=2+3 \theta_{r} / 2 .
$$

In the experiments, the $v$-integrated segment distribution is measured, which follows a scaling behavior

$$
P(z) \equiv \int_{-\infty}^{\infty} \mathrm{d} v P(z, v)=d^{-1}\left[z(d-z) / d^{2}\right]^{\theta_{r}+1 / 3} \tilde{\Omega}(z / d),
$$

where $\tilde{\Omega}(y) \equiv \int_{-\infty}^{\infty} \mathrm{d} u \Omega(y, u)$ is the corresponding shape function. The corresponding contact probability of finding a segment at $z \approx 0$ close to the wall with arbitrary tangent $v$ is

$$
\begin{aligned}
P(z) & \approx z^{1 / 3+\theta_{r}} d^{-2 \chi_{r} / 3} \tilde{\Omega}(0,0) \\
& \sim z^{2 / 3} d^{-5 / 3}
\end{aligned}
$$

\section{Appendix C. Monte Carlo simulations}

MC simulations are performed for the experimental geometries of straight and curved channels with a rectangular cross-section. We use a similar quasi-two-dimensional geometry as in the experiment with a channel height $h=1.4 \mu \mathrm{m}$ and channel widths between $d=1.5 \mu \mathrm{m}$ and $10 \mu \mathrm{m}$. For curved channels the radii of curvature are between $R=30 \mu \mathrm{m}$ and $200 \mu \mathrm{m}$. For an efficient equilibration in the simulation, we introduce a small extensibility of tangent vectors, characterized by a (large) spring constant $k$ such that the simulation model is the semiflexible harmonic chain model described in reference [33]. Introducing $N=L / b$ points $\mathbf{r}_{3, n}=\mathbf{r}_{3}(n b)(n=1, \ldots, N)$ along the chain connected by vectors $\mathbf{t}_{3, n}=\mathbf{r}_{3, n+1}-\mathbf{r}_{3, n}$, the simulated Hamiltonian is

$$
\begin{aligned}
\mathcal{H}_{3 D}= & \sum_{n=1}^{N-2} \frac{\kappa}{2 b}\left[\mathbf{t}_{3, n}-\mathbf{t}_{3, n+1}\right]^{2}+\sum_{n=1}^{N-1} \frac{k}{2}\left[\left|\mathbf{t}_{3, n}\right|-b\right]^{2} \\
& +\sum_{n=1}^{N} b V_{c h}\left(\mathbf{r}_{3, n}\right) .
\end{aligned}
$$

The discretization length $b$ has to be chosen sufficiently small not to influence results. In general, discretization effects are small in this problem. They are actually absent for a free filament without confining potential.

We use a standard Metropolis algorithm with a combination of local displacement and pivot moves of the chain. A typical MC simulation performs $\sim 10^{7} \mathrm{MC}$ sweeps over all positions $\mathbf{r}_{3, n}$. We project the three-dimensional tangent vectors $\mathbf{t}_{3, n}$ into the two-dimensional plane $y=0$ in the same manner as in the experiment, where the contour is projected into the focal plane. We measure the spatially averaged correlations of the projected tangent vectors $\mathbf{t}_{n}=\left(\mathbf{1}-\hat{\mathbf{e}}_{y} \otimes \hat{\mathbf{e}}_{y}\right) \mathbf{t}_{3, n}$, where $\hat{\mathbf{e}}_{y}$ is the unit vector in the $y$-direction. The MC algorithm that we described so far simulates ideally thin filaments. It is used to confirm that the results (1) and (2) for the tangent correlations, which were originally derived for a harmonic confining potential, remain valid for tangent correlations in steric channel confinement and to determine the appropriate value of the numerical prefactor $a \simeq 0.76$ in the Odijk scaling relation $\lambda=a L_{P}^{1 / 3} d^{2 / 3}$.

In the microscope image, however, the filament's contour has a finite thickness which cannot be neglected. The thickness originates from the finite exposure time and the limited resolution of optical microscopes. To capture these effects in the MC simulation and to allow for a detailed comparison with experimental data, we simulate the experimental imaging process within the MC simulation. Snapshots of such simulations are shown in Figure 2 along with experimental snapshots. We first mimic the experimental data acquisition process by introducing a pixel grid with a pixel size of $0.065 \mu \mathrm{m}$ comparable to the pixel size of the camera. Then we generate "microscopy images" by selecting each pixel which is positioned within a radius $R_{M C}$ of the polymer's contour at any time point during a certain MC exposure time of several hundred MC sweeps. Similar to the experimental images the radius $R_{M C}$ is much larger than the pixel size. This step leads to the loss of information about the filaments' positions on length scales smaller than $R_{M C}$. Typically we used values around $0.30 \mu \mathrm{m}$ for $R_{M C}$ which is comparable to the filament radii on the experimental binarized microscopy images. We obtain simulated binarized images as shown in Figure $2 \mathrm{~b}$. After acquiring the simulated images, we analyze them using the same procedure as for the experimental data. We first reduce the image contour which has a thickness of the order of $2 R_{M C}$ to a one-pixel-line. For this step we use the same algorithm as described in reference [34]. A cubic spline is fitted to the one-pixel-line, and an arclength reparametrization of the resulting continuous line is obtained by dividing it into tangent vectors of equal length. In the MC simulations, we can control the filament thickness $R_{M C}$ and the exposure time. As described in reference [14] this allows us to obtain much improved fits to the experimental data for tangent correlations and to accurately determine persistence and deflection lengths from these fits. 


\section{References}

1. A.R. Bausch, K. Kroy, Nature Phys. 2, 231 (2006).

2. D. Boal, Mechanics of the Cell (Cambridge University Press, Cambridge, 2002).

3. J. Howard, Mechanics of Motor Proteins and the Cytoskeleton (Sinauer, Sunderland, 2001).

4. W.W. Reisner, K.J. Morton, R. Riehn, Y.M. Wang, Z. Yu, M. Rosen, J.C. Sturm, S.Y. Chou, E. Frey, R.H. Austin, Phys. Rev. Lett. 94, 196101 (2005).

5. J.O. Tegenfeldt, C. Prinz, H. Cao, S. Chou, W.W. Reisner, R. Riehn, K.J. Morton, Y.M. Wang, E.C. Cox, J.C. Sturm, P. Silberzan, R.H. Austin, Proc. Natl. Acad. Sci. U.S.A. 101, 10979 (2004).

6. I.Y. Wong, M.L. Gardel, D.R. Reichman, E.R. Weeks, M.T. Valentine, A.R. Bausch, D.A. Weitz, Phys. Rev. Lett. 92, $178101(2004)$.

7. D. Mizuno, C. Tardin, C.F. Schmidt, F.C. MacKintosh, Science 315, 370 (2007).

8. J. Käs, H. Strey, E. Sackmann, Nature 368, 226 (1994).

9. E.G. Canty, T. Starborg, Y. Lu, S.M. Humphries, D.F. Holmes, R.S. Meadows, A. Huffman, E.T. O‘Toole, K.A. Kadler, J. Biol. Chem. 281, 38592 (2006).

10. J. Han, S.W. Turner, H.G. Craighead, Phys. Rev. Lett. 83, 1688 (1999).

11. T. Pfohl, F. Mugele, R. Seemann, S. Herminghaus, ChemPhysChem 4, 1291 (2003).

12. D. Stein, F.H.J. van der Heyden, W.J.A. Koopmans, C. Dekker, Proc. Natl. Acad. Sci. U.S.A. 103, 15853 (2006).

13. S. Köster, D. Steinhauser, T. Pfohl, J. Phys.: Cond. Mat. 17, S4091 (2005).

14. S. Köster, H. Stark, T. Pfohl, J. Kierfeld, Biophys. Rev. Lett. 2, 155 (2007).
15. M.C. Choi, C.D. Santangelo, O. Pelletier, J.H. Kim, S.Y. Kwon, Z. Wen, Y. Li, P.A. Pincus, C.R. Safinya, M.W. Kim, Macromolecules 38, 9882 (2005).

16. F. Wagner, G. Lattanzi, E. Frey, Phys. Rev. E 75, 050902(R) (2007).

17. T. Odijk, Macromolecules 16, 1340 (1983).

18. W.M. Gelbart, R.F. Bruinsma, P.A. Pincus, V.A. Parsegian, Phys. Today 53, 83 (2000).

19. T. Odijk, Macromolecules 26, 6897 (1993).

20. Y. Xia., G. Whitesides, Angew. Chem. Int. Ed. 37, 550 (1998).

21. E. Delamarche, A. Bernard, H. Schmid, H. Biebuyck, Science 276, 779 (1997).

22. J. Hendricks, T. Kawakatsu, K. Kawasaki, W. Zimmermann, Phys. Rev. E. 51, 2658 (1995).

23. G. Gompper, T.W. Burkhardt, Phys. Rev. A 40, 6124 (1989).

24. T.W. Burkhardt, J. Phys. A: Math. Gen. 26, L1157 (1993).

25. T.W. Burkhardt, J. Phys. A: Math. Gen. 30, L167 (1997).

26. J. Kierfeld, R. Lipowsky, Europhys. Lett. 62, 285 (2003).

27. M. Abramowitz, A.I. Stegun, Handbook of Mathematical Functions (National Bureau of Standards, Washington, 1965).

28. L.D. Landau, E.M. Lifshitz, Statistical Physics (Pergamon Press, London, 1958).

29. O. Kratky, G. Porod, Recl. Trav. Chim. Pay-B. 68, 1106 (1949).

30. P.G. de Gennes, Scaling Concepts in Polymer Physics (Cornell University Press, Ithaca, 1979).

31. E. Cassasaand, Y. Yagami, Macromolecules 2, 14 (1969).

32. C. Hiergeist, R. Lipowsky, Physica A 244, 164 (1997).

33. J. Kierfeld, O. Niamploy, V. Sa-yakanit, R. Lipowsky, Eur. Phys. J. E 14, 17 (2004).

34. A. Ott, M. Magnasco, A. Simon, A. Libchaber, Phys. Rev. E 48, R1642 (1993). 